\title{
On the Logarithmic Summability of Sequences in Intuitionistic Fuzzy Normed Spaces
}

\author{
Enes Yavuz \\ Department of Mathematics, Manisa Celal Bayar University, Manisa, Turkey
}

\begin{abstract}
Article Info
Keywords: Intuitionistic fuzzy normed space, Logarithmic summability, Slow oscillation, Tauberian theorem 2010 AMS: 03E72, 40A05, 40E05, $40 G 05$

Received: 10 September 2020

Accepted: 16 November 2020

Available online: 15 December 2020
\end{abstract}

\begin{abstract}
We introduce logarithmic summability in intuitionistic fuzzy normed spaces $(I F N S)$ and give some Tauberian conditions for which logarithmic summability yields convergence in IFNS. Besides, we define the concept of slow oscillation with respect to logarithmic summability in $I F N S$, investigate its relation with the concept of q-boundedness and give Tauberian theorems by means of q-boundedness and slow oscillation with respect to logarithmic summability. A comparison theorem between Cesàro summability method and logarithmic summability method in IFNS is also proved in the paper.
\end{abstract}

\section{Introduction and preliminaries}

Fuzzy sets are put forward by Zadeh [1] in 1965 as a generalization of classical sets and have been studied by many mathematicians from varied branches. In classical sets, elements in the universal set are divided crisply into two groups as members and nonmembers, and partial membership is not allowed. Unlike the classical sets, fuzzy sets allow partial membership and take every elements in the universe into account by assigning degrees of membership between 1 and 0 . Owing to the power in handling unclassifiable data, fuzzy sets are utilized in many real-world scenarios to cope with problems of uncertainty and indefiniteness. In 1983, inspired by fuzzy sets, Atanassov [2,3] considered also partial non-membership and extended fuzzy sets to intuitionistic fuzzy sets. Following Atanassov's introduction, concepts of intuitionistic fuzzy metric [4] and intuitionistic fuzzy norm ( $I F$-norm) $[5,6]$ are defined and related topics are studied. In particular, convergence of sequences in IFNS is investigated and different types of convergence(e.g., statistical convergence and ideal convergence) are applied to sequences in IFNS to grasp the convergence [7-11].

Recently Talo and Yavuz [12] introduced Cesàro summability of sequences in IFNS and gave Tauberian theorems for Cesàro summability method in IFNS, by which they initiated summability theory and Tauberian theory in IFNS. In their study, they also defined the concept of slow oscillation in IFNS and gave related theorems. Following their study, we now define logarithmic summability of sequences in IFNS and prove a Tauberian theorem for logarithmic summability method. In the sequel, we define the notion of slow oscillation with respect to logarithmic summability in IFNS and give slowly oscillating type Tauberian conditions for which logarithmic summability yields convergence in IFNS. Besides, we compare Cesàro summability and logarithmic summability in IFNS. Before continuing with main results we now give some preliminaries.

Definition 1.1. [6] The triplicate $(N, \mu, v)$ is said to be an IFNS if $N$ is a real vector space, and $\mu, v$ are fuzzy sets on $N \times \mathbb{R}$ satisfying the following conditions for every $u, w \in N$ and $t, s \in \mathbb{R}$ :

(a) $\mu(u, t)=0$ for $t \leq 0$, 
(b) $\mu(u, t)=1$ for all $t \in \mathbb{R}^{+}$if and only if $u=\theta$

(c) $\mu(c u, t)=\mu\left(u, \frac{t}{|c|}\right)$ for all $t \in \mathbb{R}^{+}$and $c \neq 0$,

(d) $\mu(u+w, t+s) \geq \min \{\mu(u, t), \mu(w, s)\}$,

(e) $\lim _{t \rightarrow \infty} \mu(u, t)=1$ and $\lim _{t \rightarrow 0} \mu(u, t)=0$,

(f) $v(u, t)=1$ for $t \leq 0$,

(g) $v(u, t)=0$ for all $t \in \mathbb{R}^{+}$if and only if $u=\theta$

(h) $v(c u, t)=v\left(u, \frac{t}{|c|}\right)$ for all $t \in \mathbb{R}^{+}$and $c \neq 0$,

(i) $\max \{v(u, t), v(w, s)\} \geq v(u+w, t+s)$,

(j) $\lim _{t \rightarrow \infty} v(u, t)=0$ and $\lim _{t \rightarrow 0} v(u, t)=1$.

We call $(\mu, v)$ an IF-norm on $N$.

Example 1.2. Let $(N,\|\cdot\|)$ be a normed space and $\mu_{0}, v_{0}$ be fuzzy sets on $N \times \mathbb{R}$ defined by

$$
\mu_{0}(u, t)=\left\{\begin{array}{ll}
0, & t \leq 0, \\
\frac{t}{t+\|u\|}, & t>0,
\end{array} \quad v_{0}(u, t)= \begin{cases}1, & t \leq 0, \\
\frac{\|u\|}{t+\|u\|}, & t>0 .\end{cases}\right.
$$

Then $\left(\mu_{0}, v_{0}\right)$ is IF-norm on $N$.

Throughout the paper $(N, \mu, v)$ will denote an IFNS.

Definition 1.3. [6] A sequence $\left(u_{n}\right)$ in $(N, \mu, v)$ is said to be convergent to $a \in N$ and denoted by $u_{n} \rightarrow a$ if for every $\varepsilon>0$ and $t>0$ there exists $n_{0} \in \mathbb{N}$ such that $\mu\left(u_{n}-a, t\right)>1-\varepsilon$ and $v\left(u_{n}-a, t\right)<\varepsilon$ for all $n \geq n_{0}$.

Definition 1.4. [6] A sequence $\left(u_{n}\right)$ in $(N, \mu, v)$ is said to be Cauchy if for every $\varepsilon>0$ and $t>0$ there exists $n_{0} \in \mathbb{N}$ such that $\mu\left(u_{k}-u_{n}, t\right)>1-\varepsilon$ and $v\left(u_{k}-u_{n}, t\right)<\varepsilon$ for all $k, n \geq n_{0}$.

Every convergent sequence is Cauchy in IFNS.

Definition 1.5. [13] A sequence $\left(u_{n}\right)$ in $(N, \mu, v)$ is called q-bounded if $\lim _{t \rightarrow \infty} \inf _{n \in \mathbb{N}} \mu\left(u_{n}, t\right)=1$ and $\lim _{t \rightarrow \infty} \sup _{n \in \mathbb{N}} v\left(u_{n}, t\right)=$ 0 .

\section{Main results}

Now we introduce logarithmic summability in IFNS and prove corresponding Tauberian theorems. For some other studies concerning logarithmic summability and convergence methods in fuzzy setting see [14-26].

Definition 2.1. Let sequence $\left(u_{n}\right)$ be in $(N, \mu, v)$. Logarithmic mean $\tau_{n}$ of $\left(u_{n}\right)$ is defined by

$$
\tau_{n}=\frac{1}{\ell_{n}} \sum_{k=1}^{n} \frac{u_{k}}{k} \quad \text { where } \quad \ell_{n}=\sum_{k=1}^{n} \frac{1}{k} .
$$

$\left(u_{n}\right)$ is said to be logarithmic summable to $a \in N$ if

$$
\lim _{n \rightarrow \infty} \tau_{n}=a .
$$

Following theorem shows that convergence yields logarithmic summability in IFNS.

Theorem 2.2. Let sequence $\left(u_{n}\right)$ be in $(N, \mu, v)$. If $\left(u_{n}\right)$ is convergent to $a \in N$, then $\left(u_{n}\right)$ is logarithmic summable to a.

Proof. Let sequence $\left(u_{n}\right)$ converge to $a \in N$. Fix $t>0$. For $\varepsilon>0$

- There exists $n_{0} \in \mathbb{N}$ such that $\mu\left(u_{n}-a, \frac{t}{2}\right)>1-\varepsilon$ and $v\left(u_{n}-a, \frac{t}{2}\right)<\varepsilon$ for $n>n_{0}$.

- There exists $n_{1} \in \mathbb{N}$ such that

$$
\mu\left(\sum_{k=1}^{n_{0}} \frac{u_{k}-a}{k}, \frac{\ell_{n} t}{2}\right)>1-\varepsilon \text { and } \quad v\left(\sum_{k=1}^{n_{0}} \frac{u_{k}-a}{k}, \frac{\ell_{n} t}{2}\right)<\varepsilon
$$

for $n>n_{1}$, since we have

$$
\lim _{n \rightarrow \infty} \mu\left(\sum_{k=1}^{n_{0}} \frac{u_{k}-a}{k}, \frac{\ell_{n} t}{2}\right)=1 \quad \text { and } \quad \lim _{n \rightarrow \infty} v\left(\sum_{k=1}^{n_{0}} \frac{u_{k}-a}{k}, \frac{\ell_{n} t}{2}\right)=0 .
$$


Hence we get

$$
\begin{aligned}
\mu\left(\frac{1}{\ell_{n}} \sum_{k=1}^{n} \frac{u_{k}}{k}-a, t\right) & =\mu\left(\frac{1}{\ell_{n}} \sum_{k=1}^{n} \frac{u_{k}-a}{k}, t\right)=\mu\left(\sum_{k=1}^{n} \frac{u_{k}-a}{k}, \ell_{n} t\right) \\
& \geq \min \left\{\mu\left(\sum_{k=1}^{n_{0}} \frac{u_{k}-a}{k}, \frac{\ell_{n} t}{2}\right), \mu\left(\sum_{k=n_{0}+1}^{n} \frac{u_{k}-a}{k}, \frac{\ell_{n} t}{2}\right)\right\} \\
& \geq \min \left\{\mu\left(\sum_{k=1}^{n_{0}} \frac{u_{k}-a}{k}, \frac{\ell_{n} t}{2}\right), \mu\left(\sum_{k=n_{0}+1}^{n} \frac{u_{k}-a}{k}, \frac{\left(\ell_{n}-\ell_{n_{0}}\right) t}{2}\right)\right\} \\
& \geq \min \left\{\mu\left(\sum_{k=1}^{n_{0}} \frac{u_{k}-a}{k}, \frac{\ell_{n} t}{2}\right), \mu\left(\frac{u_{n_{0}+1}-a}{n_{0}+1}, \frac{t}{2\left(n_{0}+1\right)}\right), \cdots, \mu\left(\frac{u_{n}-a}{n}, \frac{t}{2 n}\right)\right\} \\
& =\min \left\{\mu\left(\sum_{k=1}^{n_{0}} \frac{u_{k}-a}{k}, \frac{\ell_{n} t}{2}\right), \mu\left(u_{n_{0}+1}-a, \frac{t}{2}\right), \cdots, \mu\left(u_{n}-a, \frac{t}{2}\right)\right\} \\
& >1-\varepsilon
\end{aligned}
$$

and

$$
\begin{aligned}
& v\left(\frac{1}{\ell_{n}} \sum_{k=1}^{n} \frac{u_{k}}{k}-a, t\right)<\max \left\{v\left(\sum_{k=1}^{n_{0}} \frac{u_{k}-a}{k}, \frac{\ell_{n} t}{2}\right), v\left(u_{n_{0}+1}-a, \frac{t}{2}\right), \cdots, v\left(u_{n}-a, \frac{t}{2}\right)\right\} \\
&<\varepsilon
\end{aligned}
$$

whenever $n>\max \left\{n_{0}, n_{1}\right\}$, which completes the proof.

Logarithmic summability does not imply convergence in IFNS by the next example.

Example 2.3. Take $\left(u_{n}\right)=\left((-1)^{n}\right)$ in IF-normed space $\left(\mathbb{R}, \mu_{0}, v_{0}\right)$ where $\mu_{0}$ and $v_{0}$ are as in Example 1.2. Sequence $\left(u_{n}\right)$ is logarithmic summable to 0 in view of Theorem 2.13 and [12, Example 3.3], but it is not convergent.

We now give some Tauberian conditions for which logarithmic summability yields convergence in IFNS.

Theorem 2.4. Let sequence $\left(u_{n}\right)$ be in $(N, \mu, v)$. If $\left(u_{n}\right)$ is logarithmic summable to $a \in N$, then it converges to a if and only if for each $t>0$

$$
\sup _{\lambda>1} \liminf _{n \rightarrow \infty} \mu\left(\frac{1}{\ell_{\left\lfloor n^{\lambda}\right\rfloor}-\ell_{n}} \sum_{k=n+1}^{\left\lfloor n^{\lambda}\right\rfloor} \frac{u_{k}-u_{n}}{k}, t\right)=1
$$

and

$$
\inf _{\lambda>1} \limsup _{n \rightarrow \infty} v\left(\frac{1}{\ell_{\left\lfloor n^{\lambda}\right\rfloor}-\ell_{n}} \sum_{k=n+1}^{\left\lfloor n^{\lambda}\right\rfloor} \frac{u_{k}-u_{n}}{k}, t\right)=0
$$

Proof. Necessity. Let $\left(u_{n}\right)$ converge to $a$. For all $\lambda>1$ and large enough $n$, that is when $\left\lfloor n^{\lambda}\right\rfloor>n$, we can write(see [27, Lemma 5.5(i)])

$$
u_{n}-\tau_{n}=\frac{\ell_{\left\lfloor n^{\lambda}\right\rfloor}}{\ell_{\left\lfloor n^{\lambda}\right\rfloor}-\ell_{n}}\left(\tau_{\left\lfloor n^{\lambda}\right\rfloor}-\tau_{n}\right)-\frac{1}{\ell_{\left\lfloor n^{\lambda}\right\rfloor}-\ell_{n}} \sum_{k=n+1}^{\left\lfloor n^{\lambda}\right\rfloor} \frac{u_{k}-u_{n}}{k} .
$$

Since $\left(\tau_{n}\right)$ is Cauchy, for each $t>0$ we have

$$
\lim _{n \rightarrow \infty} \mu\left(\tau_{\left\lfloor n^{\lambda}\right\rfloor}-\tau_{n}, t\right)=1 \text { and } \lim _{n \rightarrow \infty} v\left(\tau_{\left\lfloor n^{\lambda}\right\rfloor}-\tau_{n}, t\right)=0
$$

Hence, for sufficiently large $n$ such that $\frac{\left.\ell_{\lfloor n} \lambda\right\rfloor}{\ell_{\lfloor n}{ }^{\lambda}-\ell_{n}} \leq \frac{2 \lambda}{\lambda-1}$ is satisfied, we have

$$
\mu\left(\frac{\ell_{\left\lfloor n^{\lambda}\right\rfloor}}{\ell_{\left\lfloor n^{\lambda}\right\rfloor}-\ell_{n}}\left(\tau_{\left\lfloor n^{\lambda}\right\rfloor}-\tau_{n}\right), t\right)=\mu\left(\tau_{\left\lfloor n^{\lambda}\right\rfloor}-\tau_{n}, \frac{t}{\frac{\ell_{\left\lfloor n^{\lambda}\right\rfloor}}{\ell_{\left\lfloor n^{\lambda}\right\rfloor}-\ell_{n}}}\right) \geq \mu\left(\tau_{\left\lfloor n^{\lambda}\right\rfloor}-\tau_{n}, \frac{t}{\frac{2 \lambda}{\lambda-1}}\right) \rightarrow 1 \quad(n \rightarrow \infty)
$$


104

Fundamental Journal of Mathematics and Applications

and

$$
v\left(\frac{\ell_{\left\lfloor n^{\lambda}\right\rfloor}}{\ell_{\left\lfloor n^{\lambda}\right\rfloor}-\ell_{n}}\left(\tau_{\left\lfloor n^{\lambda}\right\rfloor}-\tau_{n}\right), t\right)=v\left(\tau_{\left\lfloor n^{\lambda}\right\rfloor}-\tau_{n}, \frac{t}{\frac{\ell_{\left\lfloor n^{\lambda}\right\rfloor}}{\ell_{\left\lfloor n^{\lambda}\right\rfloor}-\ell_{n}}}\right) \leq \mu\left(\tau_{\left\lfloor n^{\lambda}\right\rfloor}-\tau_{n}, \frac{t}{\frac{2 \lambda}{\lambda-1}}\right) \rightarrow 0 \quad(n \rightarrow \infty)
$$

revealing that $\frac{\ell_{\left\lfloor n^{\lambda}\right\rfloor}}{\ell_{\left\lfloor n^{\lambda}\right\rfloor}-\ell_{n}}\left(\tau_{\left\lfloor n^{\lambda}\right\rfloor}-\tau_{n}\right) \rightarrow 0$. So, by equation (2.3), we conclude

$$
\lim _{n \rightarrow \infty} \mu\left(\frac{1}{\ell_{\left\lfloor n^{\lambda}\right\rfloor}-\ell_{n}} \sum_{k=n+1}^{\left\lfloor n^{\lambda}\right\rfloor} \frac{u_{k}-u_{n}}{k}, t\right)=1 \quad \text { and } \quad \lim _{n \rightarrow \infty} v\left(\frac{1}{\ell_{\left\lfloor n^{\lambda}\right\rfloor}-\ell_{n}} \sum_{k=n+1}^{\left\lfloor n^{\lambda}\right\rfloor} \frac{u_{k}-u_{n}}{k}, t\right)=0
$$

which means that (2.1) and (2.2) are satisfied.

Sufficiency. Let conditions (2.1) and (2.2) be satisfied. Let $t>0$ be fixed. For $\varepsilon>0$ we have:

- There exist $\lambda>1$ and $n_{0} \in \mathbb{N}$ such that

$$
\mu\left(\frac{1}{\ell_{\left\lfloor n^{\lambda}\right\rfloor}-\ell_{n}} \sum_{k=n+1}^{\left\lfloor n^{\lambda}\right\rfloor} \frac{u_{k}-u_{n}}{k}, \frac{t}{3}\right)>1-\varepsilon \quad \text { and } \quad \mu\left(\frac{1}{\ell_{\left\lfloor n^{\lambda}\right\rfloor}-\ell_{n}} \sum_{k=n+1}^{\left\lfloor n^{\lambda}\right\rfloor} \frac{u_{k}-u_{n}}{k}, \frac{t}{3}\right)<\varepsilon
$$

for $n>n_{0}$.

- There exists $n_{1} \in \mathbb{N}$ such that $\mu\left(\tau_{n}-a, \frac{t}{3}\right)>1-\varepsilon$ and $v\left(\tau_{n}-a, \frac{t}{3}\right)<\varepsilon$ for $n>n_{1}$.

- There exists $n_{2} \in \mathbb{N}$ such that

$$
\mu\left(\frac{\ell_{\left\lfloor n^{\lambda}\right\rfloor}}{\ell_{\left\lfloor n^{\lambda}\right\rfloor}-\ell_{n}}\left(\tau_{\left\lfloor n^{\lambda}\right\rfloor}-\tau_{n}\right), \frac{t}{3}\right)>1-\varepsilon \quad \text { and } \quad v\left(\frac{\ell_{\left\lfloor n^{\lambda}\right\rfloor}}{\ell_{\left\lfloor n^{\lambda}\right\rfloor}-\ell_{n}}\left(\tau_{\left\lfloor n^{\lambda}\right\rfloor}-\tau_{n}\right), \frac{t}{3}\right)<\varepsilon,
$$

$$
\text { for } n>n_{2} \text {, since } \frac{\ell_{\left\lfloor n^{\lambda}\right\rfloor}}{\ell_{\left\lfloor n^{\lambda}\right\rfloor}-\ell_{n}}\left(\tau_{\left\lfloor n^{\lambda}\right\rfloor}-\tau_{n}\right) \rightarrow 0 \text {. }
$$

Hence, by equation (2.3), we get

$$
\begin{aligned}
\mu\left(u_{n}-a, t\right) & =\mu\left(u_{n}-\tau_{n}+\tau_{n}-a, t\right) \\
& =\mu\left(\frac{\ell_{\left\lfloor n^{\lambda}\right\rfloor}}{\ell_{\left\lfloor n^{\lambda}\right\rfloor}-\ell_{n}}\left(\tau_{\left\lfloor n^{\lambda}\right\rfloor}-\tau_{n}\right)-\frac{1}{\ell_{\left\lfloor n^{\lambda}\right\rfloor}-\ell_{n}} \sum_{k=n+1}^{\left\lfloor n^{\lambda}\right\rfloor} \frac{u_{k}-u_{n}}{k}+\tau_{n}-a, t\right) \\
& \geq \min \left\{\mu\left(\frac{\ell_{\left\lfloor n^{\lambda}\right\rfloor}}{\ell_{\left\lfloor n^{\lambda}\right\rfloor}-\ell_{n}}\left(\tau_{\left\lfloor n^{\lambda}\right\rfloor}-\tau_{n}\right), \frac{t}{3}\right), \mu\left(\frac{1}{\ell_{\left\lfloor n^{\lambda}\right\rfloor}-\ell_{n}} \sum_{k=n+1}^{\left\lfloor n^{\lambda}\right\rfloor} \frac{u_{k}-u_{n}}{k}, \frac{t}{3}\right), \mu\left(\tau_{n}-a, \frac{t}{3}\right)\right\} \\
& >1-\varepsilon
\end{aligned}
$$

and

$$
\begin{aligned}
v\left(u_{n}-a, t\right) & <\max \left\{v\left(\frac{\ell_{\left\lfloor n^{\lambda}\right\rfloor}}{\ell_{\left\lfloor n^{\lambda}\right\rfloor}-\ell_{n}}\left(\tau_{\left\lfloor n^{\lambda}\right\rfloor}-\tau_{n}\right), \frac{t}{3}\right), v\left(\frac{1}{\ell_{\left\lfloor n^{\lambda}\right\rfloor}-\ell_{n}} \sum_{k=n+1}^{\left\lfloor n^{\lambda}\right\rfloor} \frac{u_{k}-u_{n}}{k}, \frac{t}{3}\right), v\left(\tau_{n}-a, \frac{t}{3}\right)\right\} \\
& <\varepsilon
\end{aligned}
$$

for $n>\max \left\{n_{0}, n_{1}, n_{2}\right\}$, which completes the proof.

Theorem 2.5. Let sequence $\left(u_{n}\right)$ be in $(N, \mu, v)$. If $\left(u_{n}\right)$ is logarithmic summable to $a \in N$, then it converges to a if and only if for each $t>0$

$$
\sup _{0<\lambda<1} \liminf _{n \rightarrow \infty} \mu\left(\frac{1}{\ell_{n}-\ell_{\left\lfloor n^{\lambda}\right\rfloor}} \sum_{k=\left\lfloor n^{\lambda}\right\rfloor+1}^{n} \frac{u_{n}-u_{k}}{k}, t\right)=1
$$

and

$$
\inf _{0<\lambda<1} \limsup _{n \rightarrow \infty} v\left(\frac{1}{\ell_{n}-\ell_{\left\lfloor n^{\lambda}\right\rfloor}} \sum_{k=\left\lfloor n^{\lambda}\right\rfloor+1}^{n} \frac{u_{n}-u_{k}}{k}, t\right)=0 .
$$


Proof. The proof is done similarly to that of Theorem 2.4 by using equation(see [27, Lemma 5.5(ii)])

$$
u_{n}-\tau_{n}=\frac{\ell_{\left\lfloor n^{\lambda}\right\rfloor}}{\ell_{n}-\ell_{\left\lfloor n^{\lambda}\right\rfloor}}\left(\tau_{n}-\tau_{\left\lfloor n^{\lambda}\right\rfloor}\right)+\frac{1}{\ell_{n}-\ell_{\left\lfloor n^{\lambda}\right\rfloor}} \sum_{k=\left\lfloor n^{\lambda}\right\rfloor+1}^{n} \frac{u_{n}-u_{k}}{k} \quad(0<\lambda<1)
$$

instead of (2.3).

Now we introduce the concept of slow oscillation with respect to logarithmic summability in IFNS.

Definition 2.6. $\left(u_{n}\right)$ in $(N, \mu, v)$ is said to be slowly oscillating with respect to logarithmic summability if

$$
\sup _{\lambda>1} \liminf _{n \rightarrow \infty} \min _{n<k \leq\left\lfloor n^{\lambda}\right\rfloor} \mu\left(u_{k}-u_{n}, t\right)=1
$$

and

$$
\inf _{\lambda>1} \limsup _{n \rightarrow \infty} \max _{n<k \leq\left\lfloor n^{\lambda}\right\rfloor} v\left(u_{k}-u_{n}, t\right)=0
$$

for each $t>0$. " $\sup _{\lambda>1}$ " in (2.4) and "inf $\lambda_{\lambda>1}$ " in (2.5) can be replaced by " $\lim _{\lambda \rightarrow 1^{+}}$".

A sequence $\left(u_{n}\right)$ in $(N, \mu, v)$ is slowly oscillating with respect to logarithmic summability if for each $t>0$ and for all $\varepsilon>0$ there exist $\lambda>1$ and $n_{0} \in \mathbb{N}$ such that

$$
\mu\left(u_{k}-u_{n}, t\right)>1-\varepsilon \text { and } \quad v\left(u_{k}-u_{n}, t\right)<\varepsilon
$$

whenever $n_{0} \leq n<k \leq\left\lfloor n^{\lambda}\right\rfloor$.

The proof of next theorem is analogous to that of Theorem 4.2 in [12] and hence omitted.

Theorem 2.7. Let sequence $\left(u_{n}\right)$ be in $(N, \mu, v)$. For $t>0$, conditions (2.4) and (2.5) are equivalent to

$$
\sup _{0<\lambda<1} \liminf _{n \rightarrow \infty} \min _{\left\lfloor n^{\lambda}\right\rfloor<k \leq n} \mu\left(u_{k}-u_{n}, t\right)=1
$$

and

$$
\inf _{0<\lambda<1} \limsup _{n \rightarrow \infty} \max _{\left\lfloor n^{\lambda}\right\rfloor<k \leq n} v\left(u_{k}-u_{n}, t\right)=0
$$

respectively. " $\sup _{0<\lambda<1}$ " in (2.6) and " $\inf _{0<\lambda<1}$ " in (2.7) can be replaced by " $\lim _{\lambda \rightarrow 1^{-}}$".

Example 2.8. Consider IF-normed space $\left(\mathbb{R}, \mu_{0}, v_{0}\right)$ where $\mu_{0}$ and $v_{0}$ are as in Example 1.2. $u_{n}=\sum_{j=1}^{n} \frac{1}{j \ln j}$ is slowly oscillating with respect to logarithmic summability by the calculations below:

Fix $t>0$. For $\varepsilon>0$ take $\lambda=e^{\frac{t \varepsilon}{1-\varepsilon}}$. Then for $1<n<k \leq\left\lfloor n^{\lambda}\right\rfloor$ we have

$$
\mu_{0}\left(u_{k}-u_{n}, t\right)=\frac{t}{t+\left|u_{k}-u_{n}\right|}>\frac{t}{t+\frac{t \varepsilon}{1-\varepsilon}}=1-\varepsilon
$$

and

$$
v_{0}\left(u_{k}-u_{n}, t\right)=\frac{\left|u_{k}-u_{n}\right|}{\left|u_{k}-u_{n}\right|+t}<\frac{\frac{t \varepsilon}{1-\varepsilon}}{\frac{t \varepsilon}{1-\varepsilon}+t}=\varepsilon
$$

since $\left|u_{k}-u_{n}\right|=\sum_{j=n+1}^{k} \frac{1}{j \ln j}<\int_{n}^{k} \frac{d u}{u \ln u} \leq \ln \left(\frac{\ln k}{\ln n}\right) \leq \ln \lambda=\frac{t \varepsilon}{1-\varepsilon}$.

Theorem 2.9. Let sequence $\left(u_{n}\right)$ be in $(N, \mu, v)$. If $\left(u_{n}\right)$ is slowly oscillating with respect to logarithmic summability then (2.1) and (2.2) are satisfied.

Proof. Suppose that $\left(u_{n}\right)$ is slowly oscillating with respect to logarithmic summability. Fix $t>0$. For $\varepsilon>0$ there exist $\lambda>1$ and $n_{0} \in \mathbb{N}$ such that

$$
\mu\left(u_{k}-u_{n}, t\right)>1-\varepsilon \quad \text { and } \quad v\left(u_{k}-u_{n}, t\right)<\varepsilon
$$


whenever $n_{0} \leq n<k \leq\left\lfloor n^{\lambda}\right\rfloor$. Hence, we have

$$
\begin{aligned}
\mu\left(\frac{1}{\ell_{\left\lfloor n^{\lambda}\right\rfloor}-\ell_{n}} \sum_{k=n+1}^{\left\lfloor n^{\lambda}\right\rfloor} \frac{u_{k}-u_{n}}{k}, t\right) & =\mu\left(\sum_{k=n+1}^{\left\lfloor n^{\lambda}\right\rfloor} \frac{u_{k}-u_{n}}{k},\left(\ell_{\left\lfloor n^{\lambda}\right\rfloor}-\ell_{n}\right) t\right) \\
& \geq \min \left\{\mu\left(\frac{u_{n+1}-u_{n}}{n+1}, \frac{t}{n+1}\right), \ldots, \mu\left(\frac{u_{\left\lfloor n^{\lambda}\right\rfloor}-u_{n}}{\left\lfloor n^{\lambda}\right\rfloor}, \frac{t}{\left\lfloor n^{\lambda}\right\rfloor}\right)\right\} \\
& =\min \left\{\mu\left(u_{n+1}-u_{n}, t\right), \ldots, \mu\left(u_{\left\lfloor n^{\lambda}\right\rfloor}-u_{n}, t\right)\right\} \\
& >1-\varepsilon
\end{aligned}
$$

and

$$
\begin{aligned}
v\left(\frac{1}{\ell_{\left\lfloor n^{\lambda}\right\rfloor}-\ell_{n}} \sum_{k=n+1}^{\left\lfloor n^{\lambda}\right\rfloor} \frac{u_{k}-u_{n}}{k}, t\right) & \leq \max \left\{v\left(u_{n+1}-u_{n}, t\right), \ldots, v\left(u_{\left\lfloor n^{\lambda}\right\rfloor}-u_{n}, t\right)\right\} \\
& <\varepsilon
\end{aligned}
$$

for $n \geq n_{0}$ and this completes the proof.

In view of Theorem 2.4 and Theorem 2.9 we give the following Tauberian theorem.

Theorem 2.10. Let sequence $\left(u_{n}\right)$ be in $(N, \mu, v)$. If $\left(u_{n}\right)$ is logarithmic summable to $a \in N$ and slowly oscillating with respect to logarithmic summability, then $\left(u_{n}\right)$ converges to a.

Theorem 2.11. Let sequence $\left(u_{n}\right)$ be in $(N, \mu, v)$. If $\left\{n \ln n\left(u_{n}-u_{n-1}\right)\right\}$ is $q$-bounded, then $\left(u_{n}\right)$ is slowly oscillating with respect to logarithmic summability.

Proof. Let $\left\{n \ln n\left(u_{n}-u_{n-1}\right)\right\}$ be q-bounded. In view of Definition 1.5, for given $\varepsilon>0$ there exists $M_{\varepsilon}>0$ so that

$$
t>M_{\varepsilon} \Rightarrow \inf _{n \in \mathbb{N}} \mu\left(n \ln n\left(u_{n}-u_{n-1}\right), t\right)>1-\varepsilon \quad \text { and } \quad \sup _{n \in \mathbb{N}} v\left(n \ln n\left(u_{n}-u_{n-1}\right), t\right)<\varepsilon .
$$

For every $t>0$ choose $\lambda<1+\frac{t}{M_{\varepsilon}}$. Then for $n_{0}<n<k \leq\left\lfloor n^{\lambda}\right\rfloor$ we have

$$
\begin{aligned}
\mu\left(u_{k}-u_{n}, t\right) & =\mu\left(\sum_{j=n+1}^{k}\left(u_{j}-u_{j-1}\right), t\right) \\
& \geq \min _{n+1 \leq j \leq k} \mu\left(u_{j}-u_{j-1}, \frac{t}{j\left(\ell_{k}-\ell_{n}\right)}\right) \\
& =\min _{n+1 \leq j \leq k} \mu\left(j \ln j\left(u_{j}-u_{j-1}\right), \frac{t \ln j}{\ell_{k}-\ell_{n}}\right) \\
& \geq \min _{n+1 \leq j \leq k} \mu\left(j \ln j\left(u_{j}-u_{j-1}\right), \frac{t \ln n}{\ell_{k}-\ell_{n}}\right) \\
& \geq \min _{n+1 \leq j \leq k} \mu\left(j \ln j\left(u_{j}-u_{j-1}\right), \frac{t}{\frac{\ln k}{\ln n}}\right) \\
& \geq \min _{n+1 \leq j \leq k} \mu\left(j \ln j\left(u_{j}-u_{j-1}\right), \frac{t}{\lambda-1}\right) \\
& \geq \inf _{n \in \mathbb{N}} \mu\left(n \ln n\left(u_{n}-u_{n-1}\right), \frac{t}{\lambda-1}\right) \\
& >1-\varepsilon
\end{aligned}
$$

and

$$
v\left(u_{k}-u_{n}, t\right)<\sup _{n \in \mathbb{N}} v\left(n \ln n\left(u_{n}-u_{n-1}\right), \frac{t}{\lambda-1}\right)<\varepsilon .
$$

Hence, $\left(u_{n}\right)$ is slowly oscillating with respect to logarithmic summability.

By Theorem 2.10 and Theorem 2.11, we conclude following Tauberian theorem. 
Theorem 2.12. Let sequence $\left(u_{n}\right)$ be in $(N, \mu, v)$. If $\left(u_{n}\right)$ is logarithmic summable to a $\in N$ and $\left\{n \ln n\left(u_{n}-u_{n-1}\right)\right\}$ is $q$-bounded, then $\left(u_{n}\right)$ converges to a.

Now we prove a comparison theorem.

Theorem 2.13. Let sequence $\left(u_{n}\right)$ be in $(N, \mu, v)$. If $\left(u_{n}\right)$ is Cesàro summable to $a \in N$, then $\left(u_{n}\right)$ is logarithmic summable to $a$.

Proof. Let $\left(u_{n}\right)$ be Cesàro summable to $a \in N$. Then, Cesàro means $\sigma_{n}=\frac{1}{n} \sum_{k=1}^{n} u_{k}$ converges to $a$ and $\frac{1}{\ell_{n}} \sum_{k=1}^{n} \frac{\sigma_{k-1}}{k} \rightarrow a$ by Theorem 2.2 with the agreement $\sigma_{0}=0$.

Fix $t>0$. For $\varepsilon>0$

- There exists $n_{0} \in \mathbb{N}$ such that $\mu\left(\sigma_{n}-a, \frac{t}{2}\right)>1-\varepsilon$ and $v\left(\sigma_{n}-a, \frac{t}{2}\right)<\varepsilon$ whenever $n>n_{0}$.

- There exists $n_{1} \in \mathbb{N}$ such that

$$
\mu\left(\frac{1}{\ell_{n}} \sum_{k=1}^{n} \frac{\sigma_{k-1}}{k}-a, \frac{t}{2}\right)>1-\varepsilon \quad \text { and } \quad v\left(\frac{1}{\ell_{n}} \sum_{k=1}^{n} \frac{\sigma_{k-1}}{k}-a, \frac{t}{2}\right)<\varepsilon
$$

whenever $n>n_{1}$.

- There exists $n_{2} \in \mathbb{N}$ such that $\mu\left(a, \frac{\left(\ell_{n}-1\right) t}{2}\right)>1-\varepsilon$ and $v\left(a, \frac{\left(\ell_{n}-1\right) t}{2}\right)<\varepsilon$ whenever $n>n_{2}$, since $\lim _{n \rightarrow \infty} \mu\left(a, \frac{\left(\ell_{n}-1\right) t}{2}\right)=$ 1 and $\lim _{n \rightarrow \infty} v\left(a, \frac{\left(\ell_{n}-1\right) t}{2}\right)=0$.

Then, we have(see [28])

$$
\begin{aligned}
\mu\left(\tau_{n}-a, t\right) & =\mu\left(\frac{\sigma_{n}}{\ell_{n}}+\frac{1}{\ell_{n}} \sum_{k=1}^{n} \frac{\sigma_{k-1}}{k}-a, t\right) \\
& \geq \min \left\{\mu\left(\frac{\sigma_{n}}{\ell_{n}}, \frac{t}{2}\right), \mu\left(\frac{1}{\ell_{n}} \sum_{k=1}^{n} \frac{\sigma_{k-1}}{k}-a, \frac{t}{2}\right)\right\} \\
& =\min \left\{\mu\left(\sigma_{n}, \frac{\ell_{n} t}{2}\right), \mu\left(\frac{1}{\ell_{n}} \sum_{k=1}^{n} \frac{\sigma_{k-1}}{k}-a, \frac{t}{2}\right)\right\} \\
& \geq \min \left\{\mu\left(\sigma_{n}-a, \frac{t}{2}\right), \mu\left(a, \frac{\left(\ell_{n}-1\right) t}{2}\right), \mu\left(\frac{1}{\ell_{n}} \sum_{k=1}^{n} \frac{\sigma_{k-1}}{k}-a, \frac{t}{2}\right)\right\} \\
& >1-\varepsilon
\end{aligned}
$$

and

$$
v\left(\tau_{n}-a, t\right) \leq \max \left\{v\left(\sigma_{n}-a, \frac{t}{2}\right), v\left(a, \frac{\left(\ell_{n}-1\right) t}{2}\right), v\left(\frac{1}{\ell_{n}} \sum_{k=1}^{n} \frac{\sigma_{k-1}}{k}-a, \frac{t}{2}\right)\right\}<\varepsilon
$$

whenever $n>\max \left\{n_{0}, n_{1}, n_{2}\right\}$, which completes the proof.

Logarithmic summability does not imply Cesàro summability in IFNS by the next example.

Example 2.14. Consider sequence $\left(u_{n}\right)=\left((-1)^{n} n\right)$ in $I F-$ normed space $\left(\mathbb{R}, \mu_{0}, v_{0}\right)$ where $\mu_{0}$ and $v_{0}$ are as in Example 1.2 . Since

$$
\begin{aligned}
& \lim _{n \rightarrow \infty} \mu_{0}\left(\tau_{2 n+1}, t\right)=\lim _{n \rightarrow \infty} \mu_{0}\left(-\frac{1}{\ell_{2 n+1}}, t\right)=\lim _{n \rightarrow \infty} \frac{t}{t+\left|-\frac{1}{\ell_{2 n+1}}\right|}=1 \\
& \lim _{n \rightarrow \infty} v_{0}\left(\tau_{2 n+1}, t\right)=\lim _{n \rightarrow \infty} v_{0}\left(-\frac{1}{\ell_{2 n+1}}, t\right)=\lim _{n \rightarrow \infty} \frac{\left|-\frac{1}{\ell_{2 n+1}}\right|}{\left|-\frac{1}{\ell_{2 n+1}}\right|+t}=0
\end{aligned}
$$

we have $\tau_{2 n+1} \rightarrow 0$, and since

$$
\lim _{n \rightarrow \infty} \mu_{0}\left(\tau_{2 n}, t\right)=\lim _{n \rightarrow \infty} \mu_{0}(0, t)=\lim _{n \rightarrow \infty} \frac{t}{t+0}=1, \quad \lim _{n \rightarrow \infty} v_{0}\left(\tau_{2 n}, t\right)=\lim _{n \rightarrow \infty} v_{0}(0, t)=\lim _{n \rightarrow \infty} \frac{0}{0+t}=0
$$

we have $\tau_{2 n} \rightarrow 0$ which yields that $\lim _{n \rightarrow \infty} \tau_{n}=0$. So, $\left(u_{n}\right)$ is logarithmic summable to 0 . But, sequence $\left(u_{n}\right)$ is not Cesàro summable. 
We note that converse of Theorem 2.13 is true under the condition $\ln n\left(\tau_{n}-a\right) \rightarrow 0$, which can be seen by the following:

$$
\begin{aligned}
\mu\left(\sigma_{n}-a, t\right) & =\mu\left(\ell_{n}\left(\tau_{n}-a\right)-\frac{1}{n} \sum_{k=1}^{n-1} \ell_{k}\left(\tau_{k}-a\right), t\right) \\
& \geq \min \left\{\mu\left(\ell_{n}\left(\tau_{n}-a\right), \frac{t}{2}\right), \mu\left(\frac{1}{n} \sum_{k=1}^{n-1} \ell_{k}\left(\tau_{k}-a\right), \frac{t}{2}\right)\right\} \rightarrow 1 \text { as } \quad n \rightarrow \infty
\end{aligned}
$$

and

$$
v\left(\sigma_{n}-a, t\right) \leq \max \left\{v\left(\ell_{n}\left(\tau_{n}-a\right), \frac{t}{2}\right), v\left(\frac{1}{n} \sum_{k=1}^{n-1} \ell_{k}\left(\tau_{k}-a\right), \frac{t}{2}\right)\right\} \rightarrow 0 \text { as } n \rightarrow \infty
$$

By Theorem 2.13 and Example 2.14, we see that logarithmic summability method is stronger than Cesàro summability method in summing up sequences in IFNS.

\section{References}

[1] L. A. Zadeh, Fuzzy sets, Inf. Control, 8 (1965), 338-353.

[2] K. Atanassov, Intuitionistic fuzzy sets, In: VII ITKR's Session, Sofia, June 1983 (Deposed in Central Sci.-Techn. Library of Bulg. Acad. of Sci., 1697/84) (in Bulgarian). Reprinted: International Journal of Bioautomation 2016; 20(S1): S1-S6 (in English).

[3] K. Atanassov, Intuitionistic fuzzy sets, Fuzzy Sets Syst., 20 (1986), 87-96.

[4] J. H. Park, Intuitionistic fuzzy metric spaces, Chaos Solitons Fractals, 22 (2004), 1039-1046.

[5] R. Saadati, J. H. Park, On the intuitionistic fuzzy topological spaces, Chaos Solitons Fractals, 27 (2006), 331-344.

[6] F. Lael, K. Nourouzi, Some results on the IF-normed spaces, Chaos Solitons Fractals, 37 (2008), 931-939.

[7] S. Karakus, K. Demirci, O. Duman, Statistical convergence on intuitionistic fuzzy normed spaces, Chaos Solitons Fractals, 35 (2008), $763-769$

[8] M. Mursaleen, S. A. Mohiuddine, On lacunary statistical convergence with respect to the intuitionistic fuzzy normed space, J. Comput. Appl. Math., $233(2009), 142-149$.

[9] M. Mursaleen, S. A. Mohiuddine, Statistical convergence of double sequences in intuitionistic fuzzy normed spaces, Chaos Solitons Fractals, 41 (2009),

[10] S. A. Mohiuddine, Q. M. Danish Lohani, On generalized statistical convergence in intuitionistic fuzzy normed space, Chaos Solitons Fractals, 42 (2009), 1731-1737.

[11] M. Mursaleen, S. A. Mohiuddine, H. H. E. Osama, On the ideal convergence of double sequences in intuitionistic fuzzy normed spaces, Comput. Math. Appl., 59 (2010), 603-611.

[12] Ö. Talo, E. Yavuz, Cesàro summability of sequences in intuitionistic fuzzy normed spaces and related Tauberian theorems, Soft Comput., (2020), doi: 10.1007/s00500-020-05301-z.

[13] H. Efe, C. Alaca, Compact and bounded sets in intuitionistic fuzzy metric spaces, Demonstr. Math., 40(2) (2007), 449-456.

[14] E. Yavuz, H. Coskun, On the logarithmic summability method for sequences of fuzzy numbers, Soft Comput., 21 (2017), 5779-5785.

[15] E. Yavuz, Tauberian theorems for statistical summability methods of sequences of fuzzy numbers, Soft Comput., 23 (2019), 5659-5665.

[16] S. A. Sezer, Logarithmic means of sequences of fuzzy numbers and a Tauberian theorem, Soft Comput., 24 (2020), 367-374.

[17] S. A. Sezer, Statistical harmonic summability of sequences of fuzzy numbers, Soft Comput., (2020), doi: 10.1007/s00500-020-05151-9.

[18] E. Dündar, Ö. Talo, F. Başar, Regularly $\left(\mathscr{I}_{2}, \mathscr{I}\right)$-convergence and regularly $\left(\mathscr{I}_{2}, \mathscr{I}\right)$-Cauchy double sequences of fuzzy numbers, International Journal of Analysis, (2013), Article ID 749684, 7 pages.

[19] E. Dündar, Ö. Talo, $\mathscr{I}_{2}$-convergence of double sequences of fuzzy numbers, Iran. J. Fuzzy Syst., 10(3) (2013), 37-50.

[20] M. R. Türkmen, E. Dündar, U. Ulusu, Fuzzy n-normlu uzaylarda çift dizilerin Lacunary ideal yakınsaklığı, International Congresson Science and Education (ICSE 2018), Afyonkarahisar, Turkey, 2018.

[21] U. Ulusu, E. Dündar, Asymptotically I-Cesàro equivalence of sequences of sets, Univers. J. Math. Appl., 1(2) (2018), 101-105.

[22] M. R. Türkmen, E. Dündar, On lacunary statistical convergence of double sequences and some properties in fuzzy normed spaces, J. Intell. Fuzzy Syst., 36(2) (2019), 1683-1690.

[23] E. Dündar, M. R. Türkmen, On $\mathscr{I}_{2}$-convergence and $\mathscr{I}_{2}^{*}$-convergence of double sequences in fuzzy normed spaces, Konuralp J. Math., 7(2) (2019), 405-409.

[24] E. Dündar, M. R. Türkmen, On $\mathscr{I}_{2}$-Cauchy double sequences in fuzzy normed spaces, Commun. Adv. Math. Sci., 2(2) (2019), 154-160.

[25] E. Dündar, M. R. Türkmen, N. P. Akın, Regularly ideal convergence of double sequences in fuzzy normed spaces, Bull. Math. Anal. Appl., 12(2) (2020), $12-26$.

[26] Ü. Totur, İ. Çanak, Tauberian theorems for ( $\bar{N} ; p ; q)$ summable double sequences of fuzzy numbers, Soft Comput., 24 (2020), 2301-2310.

[27] F. Móricz, Necessary and sufficient Tauberian conditions for the logarithmic summability of functions and sequences, Studia Math., 219 (2013), $109-121$

[28] F. Móricz, On the harmonic averages of numerical sequences, Arch. Math. (Basel), 86 (2006), 375-384. 